\title{
EVOLUCIÓN DE LAS REDES DE CATV HACIA LA TRANSMISIÓN DE DATOS
}

Grela, Abel Alejandro ${ }^{1}$

\section{RESUMEN}

Las redes de CATV (Community Antenna Television: Televisión por Antena Comunitaria) evolucionaron desde un esquema unidireccional, construidas completamente con cable coaxial, orientadas a la distribución de video analógico, a un esquema HFC (Hybrid Fibre Coaxial: Híbrido de Fibra y Coaxial), bidireccional, que posibilitó brindar servicios de datos, voz y video digitales. El estándar DOCSIS (Data Over Cable Service Interface Specification: Especificación de Interfaz para Servicios de Datos por Cable), formulado por CableLabs, fue el que tuvo mayor aceptación y permite brindar tasas de transferencia de datos cada vez mayores. SIS.

\section{INTRODUCCIÓN}

Los sistemas de CATV nacieron originalmente como redes de cable coaxial unidireccionales. Al principio eran sistemas con señales de televisión analógicas que atendían poblaciones alejadas de las plan- tas transmisoras, compartiendo el costo de la instalación de grandes antenas. El equipamiento de recepción (antenas, receptores, etc.) y acondicionamiento de señal (conversores de norma, moduladores, etc.) se encontraba en la cabecera del sistema. A todas las señales entrantes, independientemente de su origen, se les aplicaba FDM (Frequency Division Multiplexing: Multiplexación por División de Frecuencia), se amplificaban, y se transmitían en sentido descendente para distribución[1]. Con el tiempo fueron aumentando el ancho de banda de las redes desde los $220 \mathrm{MHz}$ a $330 \mathrm{MHz}, 450 \mathrm{MHz}$ y $550 \mathrm{MHz}$, a fin de sumar canales. Estas redes tenían en principio una disposición "árbol y rama", que consistía de una red troncal formada por cable coaxial de gran diámetro (para reducir las atenuaciones propias del cable) y una cascada de amplificadores para compensar la pérdida de la señal en el cable coaxial, desde la cual se derivaban, las redes de distribución formada por amplificadores de mayor ganancia y los pasivos, como ser divisores, acopladores y los taps o derivadores desde donde se conectaban los usuarios a través de cables coaxiales flexibles.

Además de ser unidireccional, las

\footnotetext{
${ }^{1}$ Departamento de Ingeniería - Facultad de Ciencias Exactas y Naturales y Agrimensura- UNNE
} 
largas cascadas de amplificadores ocasionaban ruido y eran inherentemente poco fiables $y$ propensos a fallas.

Los MSO (Multiple System Operator: Operadores de Sistemas Múltiples) de América del Norte formaron Multimedia Cable Network Partners Sistema, Ltd. (MCNS), con el propósito de definir una norma de producto y sistema capaz de proporcionar datos y servicios futuros sobre las plantas de CATV. MCNS propuso una solución basada en paquetes IP (Internet Protocol: Protocolo de Internet).

La especificación DOCSIS 1.0 resultó del esfuerzo de la MCNS y fue aceptado por unanimidad como el estándar de América del Norte, y el cuerpo del estándar fue adoptado por la SCTE (Society of Cable Telecommunications Engineers: Sociedad de Ingenieros de Telecomunicaciones por Cable) y luego por el ANSI (American National Standards Institute: Instituto $\mathrm{Na}-$ cional Estadounidense de Estándares) [2].
Termination System: Sistema de Terminación de Cable Módem) de cabecera.

CableLabs, en conjunto con las comunidades de proveedores y usuarios, definió luego DOCSIS 1.1 para el propósito de apoyar VoIP (Voice over IP: Voz sobre Protocolo de Internet) y seguridad avanzada.

Las siguientes versiones de DOCSIS: 2.0,3.0 y 3.1, fueron introduciendo mejoras tanto en seguridad, como así también en velocidades de transmisión de datos.

\section{EVOLUCIÓN DE REDES DE UNA VÍA A DOS VÍAS HFC}

La primera mejora importante a la planta de CATV fue la introducción de la tecnología de fibra óptica y el advenimiento de la planta HFC (Figura 1) [1].

Las porciones del cable coaxial y los elementos de soporte de amplificación troncal se remplazan con cable óptico multifibra

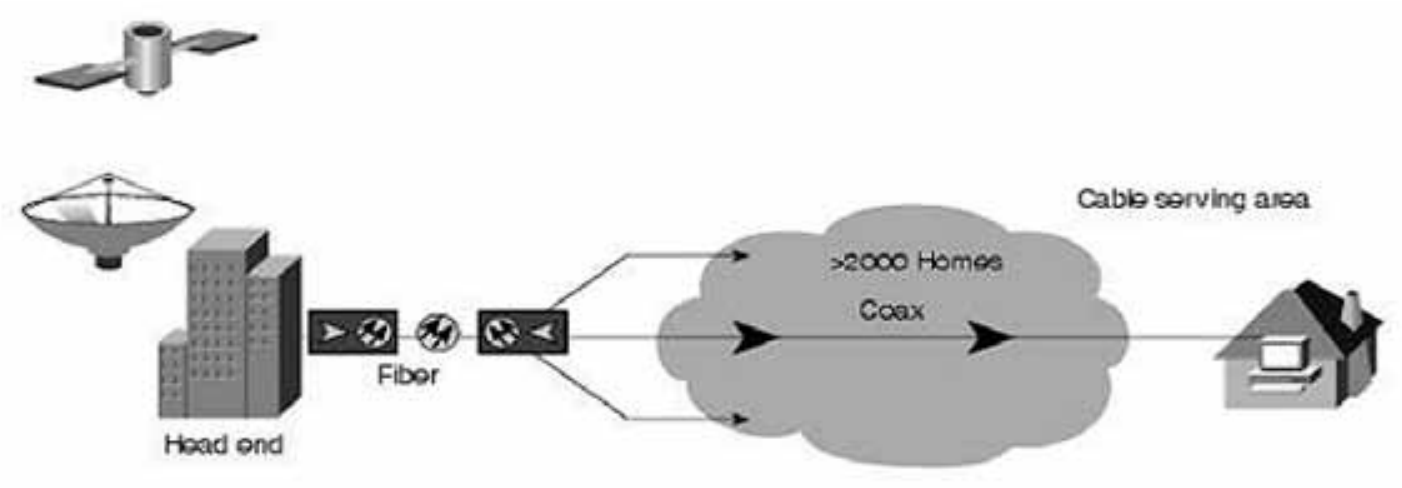

Figura 1. Red HFC [1]

El estándar DOCSIS 1.0 prescribe la interoperabilidad de múltiples proveedores y promueve un modelo de cliente de un CM (Cable Modem: Módem de Cable) y el CMTS (Cable Modem desde la cabecera. La señal de RF se utiliza para modular un láser de directa, que transmite la señal óptica a un nodo óptico, que a su vez la convierte en una señal eléctrica. La reducción del número de amplificadores 
produce una mejora la fiabilidad del sistema, la SNR (Signal to Noise Ratio: Relación Señal a Ruido) de la señal de vídeo, y el ancho de banda potencial del sistema.

El funcionamiento bidireccional se consigue mediante la adición de los amplificadores de retorno, además de un láser de retorno de banda estrecha en el nodo óptico, una fibra dedicada de retorno y, en la cabecera, un receptor óptico compatible para convertir cualquier información en una señal eléctrica.

\subsection{LA FIBRA ÓPTICA EN LAS REDES HFC}

La utilización de la fibra óptica en la distribución en principio de señales de televisión, y luego para la transmisión de datos, ha sido posible gracias al desarrollo de láseres con características de linealidad suficientes para producir distorsiones mínimas en las señales AM-VSB (Amplitude Modulation Vestigial Side Band: Modulación de Amplitud con Banda Lateral Vestigial) a transportar.

Las redes HFC, al introducir la transmisión por fibra óptica en la red de acceso, minimizan las distorsiones, introducidas por las cadenas de amplificadores.

La atenuación de la fibra es mucho menor que la del cable coaxial, lo que permite que se prolonguen las distancias a cubrir.

Una ventaja de las redes HFC es el hecho de posibilitar la transmisión de datos desde el usuario hacia la cabecera. Esto se consigue por división en frecuencia, dedicando la parte baja del espectro (de 5 a $42 \mathrm{MHz}$ típicamente) en transmisión del usuario a la cabecera.
En las redes HFC los dominios de interferencia quedan reducidos a los tramos donde se mantiene la utilización del cable coaxial, de manera que sólo comparten ancho de banda usuarios servidos por cada tramo.

Las cabeceras (o Headends) se comunican a través de fibra óptica con los nodos primarios (o Hubs), generalmente distantes de la misma y que alimentan áreas que atienden entre miles y decenas de miles de usuarios. Estos Hubs, a su vez, amplifican y distribuyen la señal hasta las ONT, dónde se realiza la conversión a la señal eléctrica que alimenta los cables coaxiales, conocidos usualmente como nodos ópticos, con uno o más receptores ópticos para la señal de directa, y uno o más transmisores ópticos para la señal de retorno.

Desde la cabecera hasta el Hub superior se dispone una fibra punto a punto.

Los sistemas ópticos troncales pueden trabajar en la segunda $(1330 \mathrm{~nm})$ o tercera $(1550 \mathrm{~nm})$ ventana óptica. Los sistemas en tercera ventana tienen la ventaja de tener alcances mayores debido a que la atenuación de la fibra a estas longitudes de onda es mínima.

$\mathrm{El}$ alcance de estos sistemas se puede aumentar con amplificación puramente óptica, motivo por el cual los nodos primarios se realizan con amplificadores ópticos de gran linealidad EDFA (Erbium Doped Fiber Amplifiers: Amplificador de Fibra Dopada con Erbio).

Por lo que respecta a los trayectos de transmisión de usuario a cabecera en la red troncal, una primera solución consiste en mantener los dominios de compartición 
del ancho de banda ascendente (y, consecuentemente, los dominios de colisión) circunscritos a cada área atendida por la red de cable coaxial, es decir, al área atendida por un nodo óptico. El canal descendente (que es el de mayor ancho de banda potencial) se comparte por todos los usuarios. Esta sencilla configuración solamente requiere instalar un transmisor óptico en sentido ascendente en el nodo óptico, un amplificador en los nodos primarios y tendidos de fibra punto a punto entre cada nodo y la cabecera.

En caso de que los Hubs atiendan a miles o decenas de miles de usuarios, la solución más adecuada y extendida en la actualidad consiste en utilizar sistemas de terminación de módems de cable (CMTS) en cada Hub, accediendo a ellos a través de una red de transmisión convencional por ejemplo, SDH (Synchronous Digital Hierarchy: Jerarquía Digital Síncrona) sobre fibra óptica, manteniendo los servidores en la cabecera. cho de banda del canal descendente se determina por los estándares de transmisión de vídeo de cada país, variando usualmente entre 6 y $8 \mathrm{MHz}$.

Las asignaciones de canales de vídeo broadcast históricos limitan el sentido ascendente o inversa (Upstream) al espectro de entre 5 y $42 \mathrm{MHz}$, aunque en la actualidad es común extenderlo a frecuencias superiores. Este espectro en sentido ascendente es a menudo hostil debido a la entrada de señales de interferencia externa como banda ciudadana, radio afición, entre otras emisiones de RF (radiofrecuencia).

El diseño, la ingeniería y las buenas prácticas de mantenimiento para las instalaciones de HFC garantizan que las especificaciones se puedan cumplir y mantener. La principal preocupación, sin embargo, se refiere al nivel de señal y el ruido.

El ancho de banda ascendente limitado debe a menudo ser compartido con

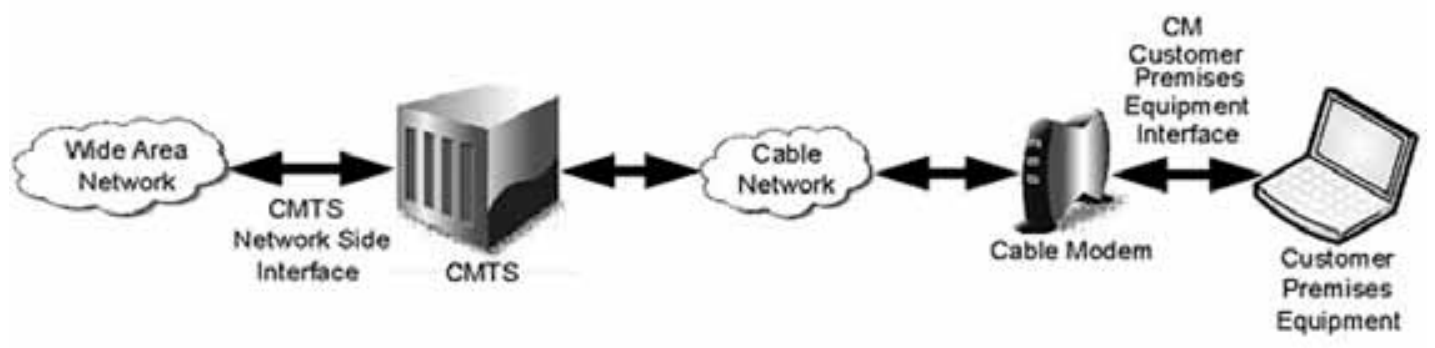

Figura 2. Tráfico IP transparente a través de DOCSIS [4]

2.2 LIMITACIONES Y ESPECIFICACIONES DE LA PLANTA HFC

$\mathrm{La}$ red HFC tiene el potencial de ofrecer gran ancho de banda en la dirección de directa (Downstream) [1]. El ancho de banda podría ser de 54 a $1002 \mathrm{MHz}$. El an- otros servicios, que van desde IPPV (Impulse Pay Per View: pago por visión por impulso), la telemetría y la recolección de alarmas e información de los elementos activos en la planta de cable.

Debido al ancho de banda ascendente limitado y a menudo hostil, el diseño del 
hardware debe implementar diversa contramedidas para mitigar los efectos de tanto perjudicial ruido fijo y transitorio.

3 Normas DOCSIS, protocolos de señalización y aplicaciones

Las especificaciones de la interfaz DOCSIS permitieron el desarrollo y el despliegue de datos por cable en un sistema no propietario, de múltiples proveedores, base interoperable para la transferencia bidireccional transparente de Internet [1]. $\mathrm{El}$ tráfico de protocolo IP entre la cabecera del sistema de cable y los usuarios se realiza mediante una red híbrida de fibra y coaxial (Figura 2). El sistema consta de un CMTS situado en la cabecera, un medio HFC, y un $\mathrm{CM}$ situado en las instalaciones del usuario final, en combinación con las capas DOCSIS definidas que admiten la interoperabilidad y permiten futuros servicios de valor agregado.

\section{Capas de DOCSIS:}

1) Capa de red IP

2) Capa de enlace de datos compuesta por:

a) Subcapa LLC (Logical Link Control: Control de Enlace Lógico) conforme a Ethernet.

b) Subcapa enlace de seguridad para la privacidad básica, autorización y autenticación.

c) Subcapa MAC (Media Access Control: Control de Acceso al Medio), para la operación de soporte de longitud variable de PDU (Protocol Data Unit: Unidades de Datos de Protocolo) y que incluyen:

I) Control de CMTS de contención de transmisión y reserva de una ráfaga de mini ranuras en el sentido ascendente.

II) La eficiencia de ancho de banda a través de paquetes de longitud variable.

III) Extensiones para el futuro apoyo de otros tipos de PDU.

IV) Soporte para múltiples grados de servicio y una amplia gama de velocidades de datos.

3) (PHY) Nivel físico compuesto por:

a) Capa de convergencia Downstream conforme a MPEG-2 (Moving Pictures Experts Group: Grupo de Expertos de Imágenes en Movimiento).

b) Subcapa PDM (Physical Medium Depedent: Física Dependiente del Medio):

I) Aguas abajo, ya sea con 64 o 256 QAM (Quadrature Amplitude Modulation: Modulación de Amplitud en Cuadratura), concatenación de Reed-Solomon y FEC (Forward Error Correction: Corrección de Errores Hacia Adelante) Trellis.

II) Aguas arriba, empleando: QPSK (Quadrature Phase Shift Keying: por desplazamiento de fase en cuadratura), 16 QAM (o actualmente 64 QAM).

III) Soporte para múltiples velocidades de símbolos CM controlada y programable desde el CMTS.

IV) Apoyo al marco fijo y formatos PDU de longitud variable.

V) TDMA (Time Division Multiple Access: Acceso Múltiple por División de Tiempo).

VI) Originalmente FEC Reed-Solomon programable. 
VII)Capacidad para soportar futuras tecnologías de capa física.

Además, la especificación define medios por los cuales un CM puede auto-descubrir las frecuencias apropiada aguas arriba $y$ aguas abajo, tasas de bits, formato de modulación, de corrección de errores, y niveles de potencia. La capa física DOCSIS permite una flexibilidad considerable para garantizar la transmisión en plantas de cable de calidad variable. Son de importancia los anchos de banda de canal aguas arriba y las opciones de modulación disponibles tanto para los flujos de señal.

En base a las opciones de ancho de banda y modulación, además de velocidades de símbolo DOCSIS especificadas existen diferentes velocidades de datos de downstream. Las tasas de datos de upstream varían según se use QPSK o QAM.

Los servidores obligatorios funcionales y operacionales deben interconectar los servicios entre el CMTS y CM.

El servidor DHCP (Dynamic Host Configuration Protocol: Protocolo de Configuración Dinámica de Cliente). Este servidor ofrece las necesarias direcciones IP tanto para el CM y PC.

El servidor TOD (Time of Day: Hora del Día), con el propósito de acotar el tiempo operativo de eventos del sistema.

El servidor TFTP (Trivial File Transfer Protocol: Protocolo de Transferencia de Archivos Trivial), con el propósito de registrar y descargar archivos de configuración para el CM. Estas configuraciones podrían incluir parámetros de QoS (Quality of Service: calidad de servicio), la implementación de BPI (Baseline Privacy Interface: Interfaz de Línea de Base de Privacidad), opera las asignaciones de frecuencia y el número de dispositivos.

Para el sentido ascendente, los operadores utilizaron en principio esquemas QPSK y luego QAM que permiten el funcionamiento dentro de un CNR (Carrier to Noise Ratio: Relación Portadora a Ruido) degradado, pero con eficiencia espectral reducida.

Además, la corrección de errores hacia delante (FEC) se puede configurar opcionalmente para reducir la cantidad de datos corrompidos por ruido. Como agregado, un ancho de banda óptimo aguas arriba, puede ser seleccionado por el operador para adaptarse a canales de datos ruidosos o espectro asignado a otros servicios. Las características físicas del hardware genérico DOCSIS 1.0, las contramedidas para mitigar el ruido, y los parámetros de la planta de cable asociado se han definido y especificado [1].

\section{Evolución de DOCSIS y capacidades de servicio}

En una planta de CATV, el CMTS podría desplegarse tanto en el Hub como en la cabecera (Headend) [1]. Además, el router de banda ancha universal puede ser equipado para conectividad de red troncal de una gran selección de adaptadores de puerto que van desde T1/E1 de serie para paquetes a través de SONET/POS (Synchronous Optical Network/ Packet Over SONET Red Óptica Síncrona/Paquetes sobre Sonet), de DPT (Transporte Dinámico de Paquetes) y Ethernet.

A menudo se requiere conectividad a PSTN (Public Switched Telephone Network: 
Red Telefónica Conmutada Pública) para apoyar a cualquiera de los servicios de acceso telefónico, a Internet o voz.

La norma evolucionó a DOCSIS 1.1, $2.0,3.0$ y 3.1 [1], para apoyar servicios adicionales y aplicaciones futuras, que coinciden con las mejoras del producto para satisfacer las necesidades del mercado, para garantizar la confiabilidad de la red, y alta disponibilidad del sistema.

Los servicios y aplicaciones actuales incluyen la telefonía basada en voz sobre protocolo de Internet (VoIP), vídeo sobre IP utilizando el formato de trama MPEG, calidad de servicio (QoS), y definiciones de seguridad mejoradas.

\subsection{DOCSIS 2.0}

La especificación DOCSIS 2.0 ofreció una tasa de transmisión de datos aguas arriba superiores por canal, aumentando el máximo a 30,72 Mbps (el triple que la norma anterior) [5].

Las funcionalidades en la vía de direc-

Tabla 8. Comparación de las caracteristicas de RF de CM hasta DOCSIS 2.0 [3]

\begin{tabular}{|c|c|c|c|}
\hline Parameter & DOCSIS 1.0 & DOCSIS 1.1 & DOCSIS 2.0 \\
\hline \multicolumn{4}{|l|}{ Downstream } \\
\hline Frecuency ( $\mathrm{MHz})$ & \multicolumn{3}{|c|}{$91-857$} \\
\hline Modulation & \multicolumn{3}{|c|}{64 QAM, 256 QAM } \\
\hline \multirow[t]{2}{*}{ Filter (eachend) } & \multicolumn{3}{|c|}{$\alpha=18 \%$ root raised cosine (64 QAM) } \\
\hline & \multicolumn{3}{|c|}{$\alpha=12 \%$ root raised cosine (256 QAM) } \\
\hline \multirow[t]{2}{*}{ Symbol rates (Mbaud) } & \multicolumn{3}{|c|}{5.0569541 (64 QAM) } \\
\hline & \multicolumn{3}{|c|}{5.360537 (256 QAM) } \\
\hline \multirow{2}{*}{$\begin{array}{l}\text { Corresponding bit rate } \\
(\mathrm{Mb} / \mathrm{s})\end{array}$} & \multicolumn{3}{|c|}{30.342 (64 QAM) } \\
\hline & \multicolumn{3}{|c|}{42.884 (256 QAM) } \\
\hline \multicolumn{4}{|l|}{ Upstream } \\
\hline Frecuency & \multicolumn{3}{|c|}{5 - $42 \mathrm{MHz}$ (65 MHz maximum, Euro DOCSIS) } \\
\hline Modulation & $\begin{array}{l}\text { QPSK (16 QAM } \\
\text { optional, rarely } \\
\text { implemented) }\end{array}$ & QPSK, 16 QAM & $\begin{array}{c}\text { Same as DOCSIS } 1.1 \\
\text { plus } 8 \text { QAM," } 32 \\
\text { QAM, * } 54 \text { QAM (S- } \\
\text { CDMA and TDMA, } \\
128 \text { QAM (S-CDMA) } \\
\text { only }\end{array}$ \\
\hline Filter & \multicolumn{3}{|c|}{$\alpha=25 \%$ root raised cosine } \\
\hline $\begin{array}{l}\text { TDMA mode symbol } \\
\text { rate (kbaud) }\end{array}$ & \multicolumn{2}{|c|}{$160,320,640,1280,2560$} & $\begin{array}{c}\text { Same as DOCSIS } 1.1 \\
\text { plus } 5120\end{array}$ \\
\hline $\begin{array}{l}\text { S-CDMA mode symbol } \\
\text { rate (kbaud) }\end{array}$ & \multicolumn{2}{|c|}{ Not applicable } & $1280.2560,5120$ \\
\hline $\begin{array}{l}\text { Channel widths }(\mathrm{kHz}) \\
\text { (1)-30 dB }\end{array}$ & \multicolumn{2}{|c|}{$200,400,800,1200,3200$} & $\begin{array}{c}\text { Sarne as DOCSIS } 1.1 \\
\text { plus } 6400\end{array}$ \\
\hline Data rates $(\mathrm{kb} / \mathrm{s})$ & $\begin{array}{c}320,640,1280 \\
2560,5120 \text { (QPSK) }\end{array}$ & $\begin{array}{l}\text { Same as DOCSIS } 1.0 \\
\text { plus } 10240 \text { (16 QAM) }\end{array}$ & $\begin{array}{c}\text { Same as DOCSIS } 1.1 \\
\text { plus up to } 30.72 \\
\mathrm{Mb} / \mathrm{s} \text { in advanced } \\
\text { modes }\end{array}$ \\
\hline IInetrasm & Nana & \& tane $[$ nnt $]$ & $24 \operatorname{tane}$ \\
\hline
\end{tabular}


ta permanecieron prácticamente sin cambios, conservando la capacidad de 64 y 256 QAM. DOCSIS 2.0 define el uso de 8-QAM, 32-QAM y adicionalmente 64QAM en retorno y los formatos de modulación de DOCSIS 1.x, y opcionalmente soporta modulación 128-QAM codificada por S-CDMA (Synchronous-Code Division Multiple Access: Acceso Múltiple por División de Código Sincrónica), incorporando spread spectrum (espectro ensanchado). migración incluso a órdenes superiores de modulación de 64 ó 256 QAM, por ejemplo 1024-QAM en directa, produciría mayor rendimiento, existen limitaciones por el hecho de que la velocidad máxima de datos en bruto hacia o desde los módems de cable es en última instancia, limitada por lo que un único gran canal de $6 \mathrm{MHz}$ puede llevar en directa, o lo que una sola campana de 6,4 $\mathrm{MHz}$ de ancho de canal puede llevar en el retorno.

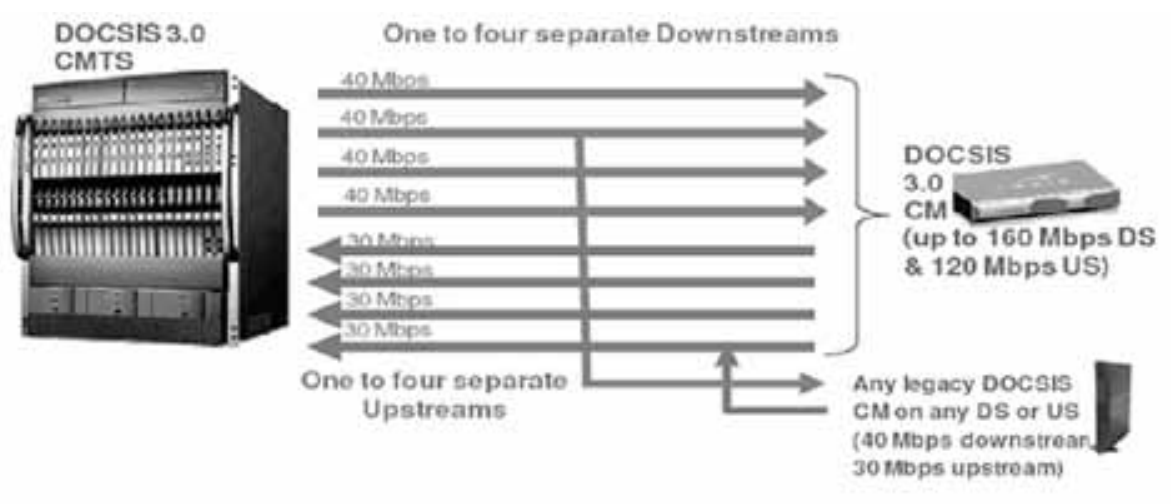

Figura 3. Channel Bonding en DOCSIS 3.0 comparado con versiones anteriores [4]

El mayor rendimiento de retorno por canal de datos disponible con la tecnología DOCSIS 2.0 se lleva a cabo utilizando órdenes superiores de modulación y el aumento de ancho de banda de canal de RF. Los órdenes de modulación más altos a QPSK y 16-QAM requieren una transmisión de datos sustancialmente más robusta. Esto es especialmente necesario en el limitado y hostil espectro inverso de RF utilizado en la mayoría de las redes de cable.

Para facilitar la transmisión más robusta de datos en sentido ascendente, DOCSIS 2.0 introdujo una serie de características llamadas PHY avanzada. A pesar de las mejoras que se han producido en la evolución de DOCSIS, las tasas máximas de datos hacia y desde el módems tienen en esta versión más o menos los límites anteriores (la comparación con las versiones anteriores se muestran en la Tabla 1 ). Mientras la

\subsection{DOCSIS 3.0}

Mientras que DOCSIS 1.0, 1.1 y 2.0, utilizan un único canal para transportar datos de directa y otro para los datos de retorno, la especificación DOCSIS 3.0 incluye la unión de canales (channel bonding) tanto en directa como en retorno; y una serie de mejoras, como ser el soporte a IPv6 [5].

La unión de canales proporciona una forma flexible para aumentar el rendimiento, con velocidades de datos en potencialmente gigabits por segundo. En pocas palabras, la unión de canales significa que los datos se transmiten desde o hacia los módems múltiples canales de RF en lugar de un solo canal (Figura 3).

Los canales no están unidos físicamente en una gigantesca señal digitalmente modulada. Más bien, la unión es lógica. Si se 
desea aumentar la velocidad de datos aguas abajo entre el CMTS y módems de hoy, el único límite es el canal de $6 \mathrm{MHz}$ de ancho de 42,88 Mbps. Si se van a utilizar datos de bajada a través de cuatro canales de $6 \mathrm{MHz}$, la velocidad de datos combinada utilizando 256-QAM en cada canal sería 42,88 Mbps x 4 = 171,52 Mbps. Un módem DOCSIS 3.0 incorporará un sintonizador especial capaz de recibir simultáneamente datos de los cuatro canales. Para el módem, los cuatro canales son el equivalente lógico de una gran canal de servicio, a pesar de que se utilizan cuatro canales separados físicamente. Con 10 canales, se producirán 42,88 Mbps x $10=428,8 \mathrm{Mbps}$, y con la unión de 24 canales se convierten en $24 \times 42.88 \mathrm{Mbps}=$ 1,029.12 Mbps, o un poco más de 1 Gbps. [5]

El mismo concepto de unión de canales es aplicable al retorno, que nos da la capacidad de ir más allá del límite de DOCSIS 2.0 por canal de 30.72 Mbps.

Hasta la versión 3.0 del estándar es muy sentido una gran ayuda la constituyen los sistemas de pre ecualización de la señal, que pre distorsionan la misma para adaptarla a las alinealidades del canal de comunicación.

\subsection{DOCSIS 3.1}

Para obtener mayor eficiencia espectral DOCSIS 3.1 trabaja con modulaciones de mayor orden [6]. Las modulaciones de mayor orden son más eficientes pero a la vez más vulnerables al ruido e interferencia.

Para mejorar la robustez se trabaja con OFDM (Orthogonal Frequency Division Multiplexing: Multiplexación por División de Frecuencias Ortogonales) y además se implementa corrección de errores por LDPC (Low Density Parity Check: Comprobación de Paridad de Baja Densidad). En este sentido se utilizan múltiples subportadoras espaciadas $20 \mathrm{KHz}$ a $50 \mathrm{KHz}$, cada sub-portadora se controla en forma independiente pudiendo encenderse o apagarse, controlar su nivel y cambiar el orden

Tabla 2. Capacidad Actuales y Futuras - DOCSIS 3.0 y 3.1 [6]

\begin{tabular}{lcccc}
\hline & Actual & Futuro 1 & Futuro 2 & Futuro 3 \\
\hline Versión de DOCSIS & 3.0 & 3.0 & 3.0 & 3.1 \\
\hline Rango de Downstream & $54-860$ & $54-1002$ & $80-1002$ & $300-1200$ \\
\hline $\begin{array}{l}\text { Modulación de } \\
\text { Downstream }\end{array}$ & 256 & 256 & 256 & $\geq 1024$ \\
\hline $\begin{array}{l}\text { Dowstream Bonding } \\
\text { Capacidad Downstream }\end{array}$ & $160 \mathrm{M}$ & $1 \mathrm{G}$ & $1,25 \mathrm{G}$ & $3,8 \mathrm{G}$ \\
\hline Rango Upstream & $5-42$ & $5-42$ & $5-65$ & $5-230$ \\
\hline $\begin{array}{l}\text { Modulación de } \\
\text { Upstream }\end{array}$ & 16 & 64 & 64 & $\geq 256$ \\
\hline $\begin{array}{l}\text { Upstream Bonding } \\
\text { Capacidad Upstream }\end{array}$ & 2 & 4 & 8 & 32 \\
\hline
\end{tabular}

importante el mantenimiento de la red, sobre todo en el espectro de retorno. En este de modulación. Con ello se logra mayor eficiencia espectral que con channel bonding y permite operar con canales de $192 \mathrm{MHz}$. 


\section{Conclusiones y líneas futuras de tra- bajo}

Las redes de difusión coaxial cuentan con limitaciones propias a su arquitectura en cuanto a la calidad de la señal de directa y se vuelve prácticamente inviable para la transmisión en doble vía, dado que el espectro de menor frecuencia comúnmente usado para el retorno sufre las consecuencias del ruido, las distorsiones y la sumatoria de usuarios de toda la red.

Las redes HFC brindan la posibilidad de construir redes bidireccionales, en función de la utilización de la fibra óptica y la reducción de las áreas de servicio.

El estándar DOCSIS, junto a protocolos de señalización, servidores necesarios, y especificaciones de los productos genéricos permiten una utilización eficaz de la planta HFC.

La evolución de DOCSIS permite ampliar las posibilidades actuales a niveles superiores en el futuro, los que están resumidos en la Tabla 2.

En este sentido se comienza a utilizar
[1] Cisco, Cable Access Technologies, 2009.

[2] Ciciora,Walter; Farmer,James; Large, Davis; Adams, Michael; Modern Cable Television Technology,Video,Voice,AndDataCommunications; San Francisco, 2004, Elsevier Inc.

[3] CableLabs, Data-Over-Cable Service Interface Specifications DOCSIS $^{\circledR}$ 3.1, Physical Layer Specification, CM-SP-PHYv3.1-I03-140610, 2014.

[4] Al-Banna, Ayham; Allen, Jim; Cloonan, Tom; DOCSIS 3.0 Upstream Channel un nuevo dispositivo que facilita la evolución hacia una arquitectura de acceso a la red convergente, llamado CCAP (Converged Cable Access Plataform: Plataforma de Acceso de Cable Convergente) [7].

Este nuevo equipo integra las funciones de QAM de broadcast y narrowcast, así como también las interfaces de Upstream y Downstream.

Así mismo se prevé la inclusión del soporte de terminales de Redes PON (Passive Optical Network: Redes Pasivas Ópticas) en el mismo chasis.

La red HFC logra un compromiso entre el coste y las prestaciones, limitando el uso de la fibra óptica hasta un nodo óptico, que atiende a varios usuarios, con un suficiente ancho de banda para la gran mayoría de las necesidades de tasas de transferencia de datos actuales y futuras.

La desaparición de la transmisión de señales de televisión analógica y la liberación de unos $500 \mathrm{MHz}$ de ancho de banda permitirá su utilización en la transmisión de datos que se requieren en los nuevos sistemas basados en las últimas versiones de DOCSIS.

Bonding: Performance Analysis in the presence of HFC Noise, SCTE, 2009.

[5] Hranac Ron; DOCSIS 3.0, SCTE Technical Columns, 2006.

[6] García Bish, Juan Ramón; Nuestras Redes de Cable, están listas para Docsis 3.1?, Encuentro Regional de Telecomunicaciones, Rosario, 2014.

[7] Martínez, Hernán D., Conceptos y Ventajas de Emigrar a DOCSIS 3.0, CNA Cable Networks, ATVC, 2011. 